ARTICLE

\title{
Lead compounds for the development of SARS- CoV-2 3CL protease inhibitors
}

Sho Iketani (10 1,2, Farhad Forouhar ${ }^{3}$, Hengrui Liu (1) ${ }^{4}$, Seo Jung Hong ${ }^{5}$, Fang-Yu Lin (10 6, Manoj S. Nair (1) ${ }^{1}$, Arie Zask ${ }^{7}$, Yaoxing Huang $\mathbb{D}^{1}{ }^{1}$, Li Xing $\mathbb{D}^{6}{ }^{6}$, Brent R. Stockwell ${ }^{4}{ }^{4,7 凶}$, Alejandro Chavez (i) ${ }^{5 凶} \&$ David D. Ho (i) ${ }^{1,2}$ 鴈

We report the identification of three structurally diverse compounds - compound 4, GC376, and MAC-5576 - as inhibitors of the SARS-CoV-2 3CL protease. Structures of each of these compounds in complex with the protease revealed strategies for further development, as well as general principles for designing SARS-CoV-2 $3 \mathrm{CL}$ protease inhibitors. These compounds may therefore serve as leads for the basis of building effective SARS-CoV-2 $3 \mathrm{CL}$ protease inhibitors.

\footnotetext{
${ }^{1}$ Aaron Diamond AIDS Research Center, Columbia University Irving Medical Center, New York, NY, USA. ${ }^{2}$ Department of Microbiology and Immunology, Columbia University Irving Medical Center, New York, NY, USA. ${ }^{3}$ Herbert Irving Comprehensive Cancer Center, Columbia University Irving Medical Center, New York, NY, USA. ${ }^{4}$ Department of Chemistry, Columbia University, New York, NY, USA. ${ }^{5}$ Department of Pathology and Cell Biology, Columbia University Irving Medical Center, New York, NY, USA. ${ }^{6}$ WuXi AppTec, Cambridge, MA, USA. ${ }^{7}$ Department of Biological Sciences, Columbia University, New York, NY, USA. email: bstockwell@columbia.edu; ac4304@cumc.columbia.edu; dh2994@cumc.columbia.edu
} 
A $s$ the etiologic agent of COVID-19, SARS-CoV-2 has resulted in millions of deaths and caused rampant economic damage worldwide ${ }^{1,2}$. While some treatments have been identified, their clinical efficacy is low or require delivery within a narrow treatment window, making continued research for additional therapeutics essential ${ }^{3,4}$. Similar to other coronaviruses, SARS-CoV-2 encodes an essential 3CL protease ( $3 \mathrm{CL}^{\text {pro }}$ or $\mathrm{M}$ pro) that processes its polyproteins, which has garnered interest as a target for potential viral inhibitors ${ }^{5,6}$. Here, we describe a series of compounds with inhibitory activity against SARS-CoV-2 3CL ${ }^{\text {pro }}$ and determine their structures in complex with the protease. These data provide general insights into the design of 3CL protease inhibitors, along with potential avenues by which these classes of compounds can be further developed.

\section{Results}

Identification of SARS-CoV-2 3CL protease inhibitors. We hypothesized that previously identified SARS-CoV 3CL protease inhibitors may also be effective against the SARS-CoV-2 3CL, given the conservation between the two proteases (96\% amino acid identity $)^{1,2}$. To study such compounds, we first purified the native SARS-CoV-2 3CL protease from Escherichia coli and confirmed that it had functional enzymatic activity in an in vitro biochemical assay (Fig. 1a, b). Using this assay to report SARSCoV-2 3CL protease activity, we identified three diverse compounds of interest: compound $4^{7}, \mathrm{GC}_{3} 76^{8}$, and MAC-5576 ${ }^{9}$ (Fig. 2a). These compounds demonstrated inhibition of the protease with $\mathrm{IC}_{50}$ values (mean \pm s.e.m.) of $151 \pm 15,160 \pm 34$, and $81 \pm 12 \mathrm{nM}$, respectively (Fig. 2b). We further characterized these compounds by conducting enzyme kinetic studies to determine the inactivation rate $\left(k_{\text {inact }} / K_{\mathrm{i}}\right)$ for each compound (Fig. 2c). Compound 4 had a $k_{\text {inact }} / K_{\mathrm{i}}$ of $4.13 \times 10^{5} \mathrm{M}^{-1} \mathrm{~s}^{-1}$ and GC376 had a $k_{\text {inact }} / K_{\mathrm{i}}$ of $6.18 \times 10^{6} \mathrm{M}^{-1} \mathrm{~s}^{-1}$, but we did not observe time-dependent inhibition by MAC-5576.

We then tested these compounds for inhibition of SARS-CoV2 viral replication. We found that compound 4 and GC376 could block viral infection in Vero-E6 cells in a cytopathic effect reduction assay $\left(\mathrm{EC}_{50}\right.$ values (mean \pm s.d.): $2.88 \pm 0.23$ and $2.19 \pm$ $0.01 \mu \mathrm{M}$, respectively), whereas MAC-5576 did not (Fig. 2d). We confirmed that these compounds did not result in cytotoxicity to the cells at the tested concentrations (Supplementary Fig. 1).
Crystal structures of $3 C^{\text {pro }}$ with protease inhibitors. As the three compounds exhibited inhibitory activity against the SARSCoV-2 3CLpro, we proceeded to solve the crystal structure of the ligand-free 3CL protease alone and of each of these compounds in complex with the protease to understand their mechanism of binding as well as to guide future structure-based optimization efforts. We note that while MAC-5576 did not exhibit activity in the cellular assay, its low molecular weight and reasonable biochemical activity prompted us to pursue its crystallization as well, as our goal was to broadly investigate inhibitory scaffolds for the SARS-CoV-2 3CL protease. Crystals were obtained (see Methods for detailed information) and structures at 1.85, 1.94, 1.83, $1.73 \AA$ resolution limits for ligand-free $3 \mathrm{CL}^{\text {pro }}$ and $3 \mathrm{CL}$ pro bound to compound 4, GC376, and MAC-5576, respectively, were solved (Fig. 2, see Table 1 for statistics).

The X-ray crystal structures revealed that all three compounds bind covalently to the catalytically active Cys 145 residue within the substrate-binding pocket of the protease. We observed distinct mechanisms by which these compounds acted on this residue. Compound 4 functioned in a similar binding mode as other reported compounds, covalently modifying Cys145 through Michael addition (Fig. 3a) ${ }^{5}$. For GC376, the bisulfite adduct was converted to an aldehyde as previously reported, allowing it to then react with Cys145 through nucleophilic addition and hemithioacetal formation (Fig. 3b) ${ }^{8}$. MAC-5576 also covalently modified Cys145 by nucleophilic linkage, which was somewhat unexpected, given that we did not observe time-dependent inhibition by this compound (Fig. 3c). We observed weaker density in the S4 site for compound 4 (Fig. 3d) and in the S3 site for GC376 (Fig. 3e) as compared to other regions of each inhibitor. For MAC-5576, we found that the overall electron density is weak, and that the optimal modeling was achieved when the occupancy was set to 0.5 , supporting that it may bind reversibly (Fig. 3f).

Structural insights into the design of 3CL protease inhibitors. As we solved the structures for multiple compounds, we hypothesized that general principles for the design of SARS-CoV-2 3CL protease inhibitors could be identified. We first overlaid all four crystal structures of the $3 \mathrm{CL}^{\text {pro }}$ with or without inhibitors (Fig. 4a). We observed local conformational changes, with Thr45

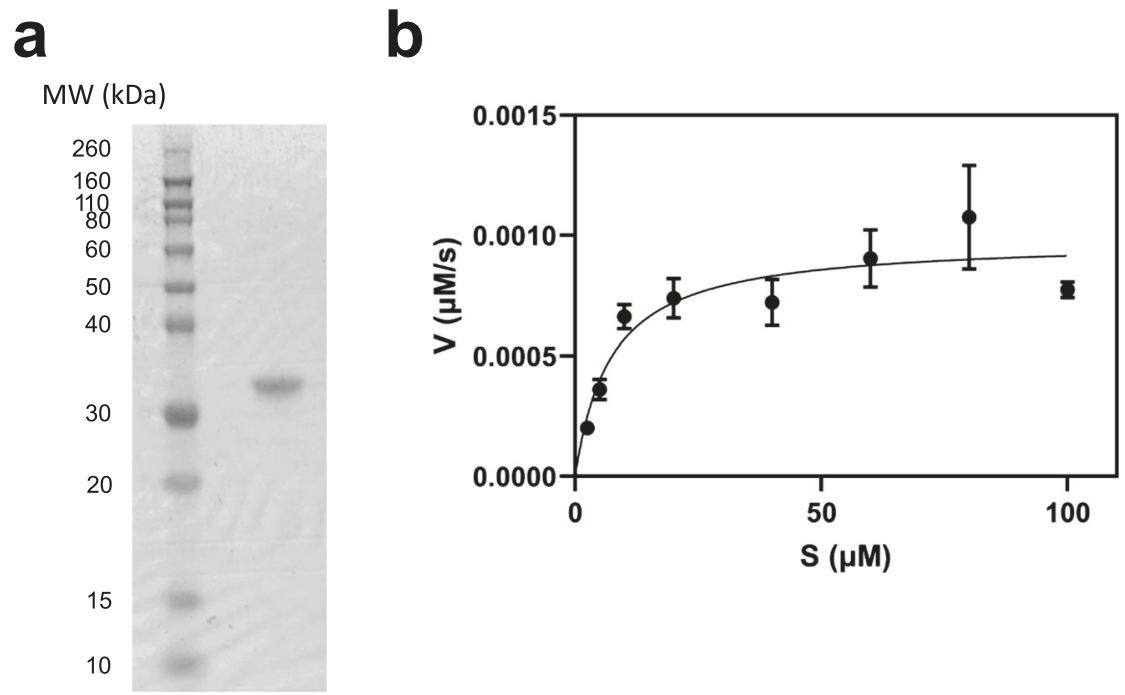

Fig. 1 Production of native SARS-CoV-2 3CL protease in E. coli. a The purified protease was ran on SDS-PAGE to confirm size and purity. $\mathbf{b}$ Confirmation of enzymatic activity of SARS-CoV-2 $3 \mathrm{CL}$ protease by quantification of cleavage of a fluorogenic peptide substrate. $K_{\mathrm{m}}$ was $7.22 \pm 2.48 \mu \mathrm{M}, V_{\text {max }}$ was $1 \pm$ $0.08 \mathrm{nM} / \mathrm{s}$, and catalytic efficiency $\left(k_{\mathrm{cat}} / K_{\mathrm{m}}\right)$ was $6,925 \mathrm{M}^{-1} \mathrm{~s}^{-1}$. Data are shown as mean \pm s.e.m. for three independent biological replicates. MW molecular weight. 
a
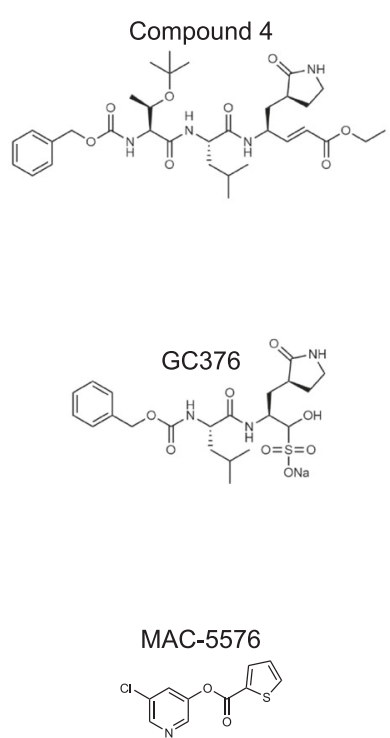

b
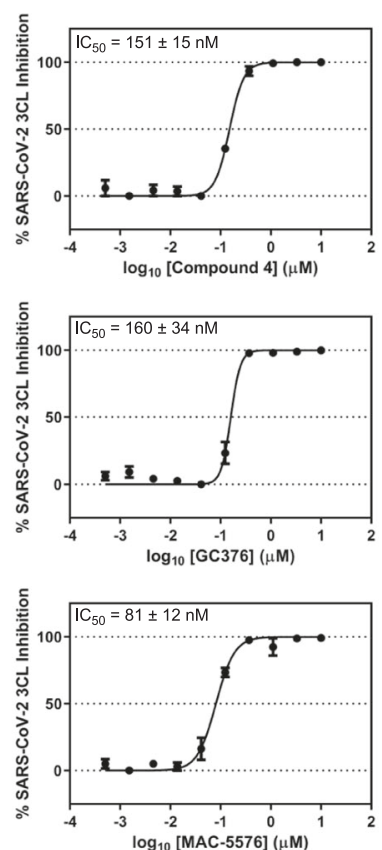

c
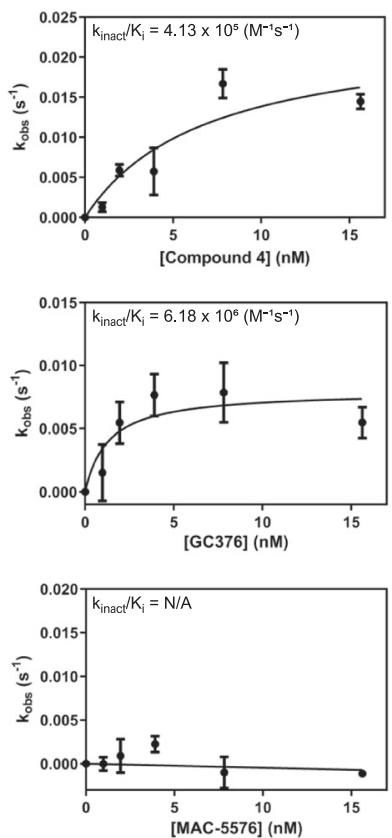

d
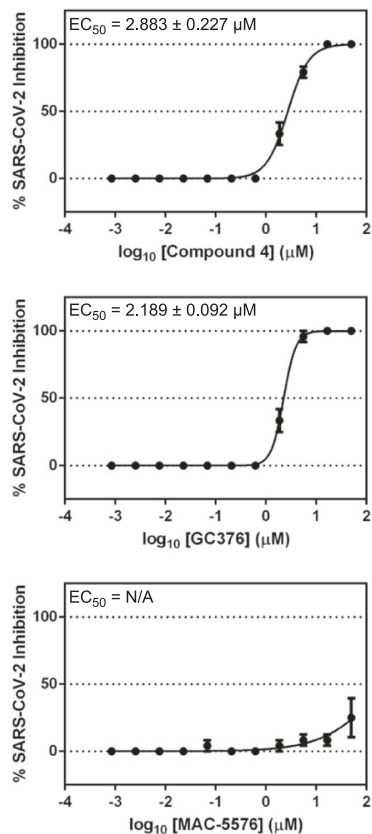

Fig. 2 Inhibition of SARS-CoV-2 3CL protease by compound 4, GC376, and MAC-5576. a Chemical structures of the three compounds in this study. b Inhibition of purified native SARS-CoV-2 3CL protease by each compound. c Kinetics of inhibition of SARS-CoV-2 3CL protease by each compound. d Inhibition of SARS-CoV-2 viral replication by each compound. Data are shown as mean \pm s.e.m. for two or three technical replicates for $\mathbf{b}$, two technical replicates for $\mathbf{c}$, and for three technical replicates for $\mathbf{d}$. $\mathrm{IC}_{50}$ and $\mathrm{EC}_{50}$ values denote mean \pm s.e.m. for two independent biological replicates.

to Pro52 distinct from the ligand-free 3CLpro in all three inhibitor-bound structures, whereas Arg188 to Gln192 differed only in the compound 4 and GC376-bound, but not MAC-5576bound structures. We then overlaid each of the inhibitors in the substrate-binding pocket of the 3CL protease to find commonalities in their interactions (Fig. 4b). Most notably, we found that all of these compounds occupied the S2 site, with compound 4 and GC376 further anchored in the S1 site. The backbone NH of Gly143 points toward the ligand-binding pocket, forming hydrogen bonds with the carbonyl oxygen of the ethyl ester of compound 4, and the hemithioacetal of GC376 after the Cys145 addition to the original aldehyde, even though the former hydrogen bond is stronger than the latter. In both structures, the $\gamma$-lactam groups occupy the S1 site, and are strongly anchored by two hydrogen bonds with the side chains of His163 and Glu166. The isobutyl groups are favorably embedded in the hydrophobic S2 site, surrounded by the alkyl portion of the side chains of His41, Met49, His164, Met165, Asp187, and Gln189. Extending into the S3 pocket, the amide bonds of compound 4 and GC376 are stabilized by hydrogen bond interactions with the side chain of $\mathrm{Gln} 189$.

To further study the interaction of compound 4 with SARSCoV-2 3CLpro, we determined and refined an additional crystal structure of the 3CL protease in complex with compound 4 in space group $P 1$ at $1.84 \AA$ resolution limits, in which there are four protomers in the asymmetric unit of the crystal, which is equivalent with the unit cell in this space group. Overlaying these protomers revealed that in particular, compound 4 exhibited significant flexibility in the $S 1^{\prime}$ region (Fig. 4c).

As several SARS-CoV-2 3CL protease inhibitors have been reported, we overlaid compound 4 and GC376 with these related substrate mimetic inhibitors (Fig. 4d) $)^{5,6,10}$. We found similar interactions between these compounds, suggesting that overall, the binding modes of this class share remarkable similarities. Specifically, they all have a $\gamma$-lactam occupying the S1 pocket, preserving the dual hydrogen bonds with His163 and Glu166. Furthermore, they commonly contain a hydrophobic moiety occupying the S2 site. The segment of the inhibitors from S1 to S2 align closely on top of each other. Variations of binding start to emerge in the S3 and S4 region, which exhibits high degrees of freedom in terms of structural diversity as well as conformational flexibility.

On the other hand, the binding of MAC-5576, as a nonpeptidic small molecule, displays unique features that differ from that of compound 4 or GC376. We observed that the thiophene group forms $\pi-\pi$ stacking with the His41 side chain imidazole, which undergoes a conformational rotation around its betacarbon to align parallel to the thiophene, as compared to the other peptide-bound structures. Additionally, the side chain of Gln189 also shows notable conformational variation compared to those in the compound 4 and GC376 crystal structures, possibly in response to the specific hydrogen bond interactions induced by the respective ligands. Notably, the rotation of His41 has been reported previously in the crystal structure of a benzotriazole ester inhibitor (XP-59) in complex with the SARS-CoV 3CL protease $(\mathrm{PDB}: 2 \mathrm{~V} 6 \mathrm{~N})^{11}$. An overlaid model of the crystal structures of MAC-5576 bound to SARS-CoV-2 3CLpro and XP-59 bound to SARS-CoV 3CLpro shows that both compounds have similar binding modes when covalently bound to Cys145, in which the thiophene of MAC-5576 and the phenyl ring of XP-59 almost overlap with each other, both engaging the His41 side chain via $\pi-\pi$ stacking interactions (Fig. $4 \mathrm{e}$ ).

\section{Discussion}

In this study, we have identified compound 4, GC376, and MAC5576 as inhibitors of the SARS-CoV-2 3CL protease. Each of these compounds displayed biochemical inhibition of the protease, and compound 4 and GC376 also inhibited the virus in a cell-based assay, whereas MAC-5576 did not (Fig. 2). We solved the crystal structures of these compounds complexed to the 
Table 1 Data collection and refinement statistics of ligand-free SARS-CoV-2 3CL protease and 3CL protease bound to compound 4, GC376, and MAC-5576.

\begin{tabular}{|c|c|c|c|c|c|}
\hline Data collection & $\begin{array}{l}\text { Ligand-free 3CL } \\
\text { (PDB: 7JST) }\end{array}$ & $\begin{array}{l}\text { 3CL with compound } 4 \\
\text { (PDB: 7JT7) }\end{array}$ & $\begin{array}{l}\text { 3CL with compound } 4 \\
\text { (PDB: 7JW8) }\end{array}$ & $\begin{array}{l}\text { 3CL with GC376 } \\
\text { (PDB: 7JSU) }\end{array}$ & $\begin{array}{l}\text { 3CL with MAC-5576 } \\
\text { (PDB: 7JTO) }\end{array}$ \\
\hline Space group & $C 2$ & $C 2$ & $P 1$ & $C 2$ & $C 2$ \\
\hline \multicolumn{6}{|l|}{ Cell dimensions } \\
\hline$\alpha, \beta, \gamma\left({ }^{\circ}\right)$ & $90,114.9,90$ & $90,117.1,90$ & $75.2,79.3,67.9$ & $90,114.3,90$ & $90,114.83,90$ \\
\hline Resolution $(\AA)$ & $60.4-1.85(1.88-1.85)^{a}$ & $59.5-1.94(1.98-1.94)^{a}$ & $90.03-1.84(1.86-1.84)^{a}$ & $59.9-1.83(1.86-1.83)^{a}$ & $60.58-1.73(1.76-1.73)^{a}$ \\
\hline$R_{\text {merge }}(\%)$ & $7.2(65.7)$ & $18.2(58.4)$ & $13.2(76.5)$ & $14.7(60.5)$ & $4.1(68.8)$ \\
\hline Redundancy & $6.8(6.5)$ & $6.7(6.5)$ & $3.6(3.1)$ & $6.9(6.1)$ & $6.8(6.6)$ \\
\hline $\mathrm{CC} 1 / 2$ & $0.99(0.95)$ & $0.99(0.92)$ & $0.99(0.89)$ & $0.99(0.93)$ & $1.00(0.90)$ \\
\hline \multicolumn{6}{|l|}{ Refinement } \\
\hline Resolution $(\AA)$ & $47.0-1.85(1.88-1.85)^{a}$ & $48.20-1.94(1.98-1.94)^{a}$ & $90.03-1.84(1.86-1.84)^{a}$ & $\begin{array}{l}47.38-1.83 \\
(1.86-1.83)\end{array}$ & $47.03-1.73(1.75-1.73)$ \\
\hline No. reflections & 31,709 (3219) & $27,502(2673)$ & $113,161(11,324)$ & $31,971(3134)$ & $38,879(3899)$ \\
\hline Allowed & 1.00 & 1.64 & 1.81 & 1.30 & 2.00 \\
\hline Favored & 99.00 & 98.36 & 98.02 & 98.70 & 98.00 \\
\hline \multicolumn{6}{|l|}{ No. atoms } \\
\hline Protein & 2329 & 2347 & 9452 & 2340 & 2317 \\
\hline Ligand/ion & 5 & 45 & 208 & 39 & 12 \\
\hline Water & 128 & 274 & 1048 & 183 & 241 \\
\hline \multicolumn{6}{|l|}{ B-factors } \\
\hline Protein & 50.0 & 32.5 & 29.6 & 40.5 & 41.6 \\
\hline Ligand/ion & 52.6 & 32.1 & 33.8 & 40.0 & 41.1 \\
\hline Water & 53.5 & 41.5 & 40.0 & 47.9 & 49.9 \\
\hline \multicolumn{6}{|l|}{ R.m.s deviations } \\
\hline Bond lengths $(\AA)$ & 0.006 & 0.006 & 0.006 & 0.006 & 0.006 \\
\hline Bond angles $\left({ }^{\circ}\right)$ & 0.8 & 0.8 & 0.8 & 0.8 & 0.8 \\
\hline
\end{tabular}

protease, confirming that each are covalent inhibitors (Fig. 3). Compound 4 and GC376 demonstrated similar interactions as other substrate mimetic inhibitors ${ }^{5,6,10}$, and MAC-5576 was similar to a previously identified small molecule inhibitor of SARS-CoV 3CLpro 11 (Fig. 4).

GC376 has been recently reported to be an inhibitor of the SARS-CoV-2 3CL pro, and the complex was solved by Ma et al. $(\mathrm{PDB} \text { accession code } 6 \mathrm{WTT})^{12}$. Our results corroborate their findings, and we observe similar interactions in our solved structure. However, one notable difference lies in the S3 site, in which the benzyl group in our crystal structure points upward towards the solvent, while making a hydrophobic interaction with the lactam group. In contrast, the benzyl group of GC376, bound to each of the three $3 \mathrm{CL}$ protomers in the asymmetric unit (ASU) of their structure, is anchored in the hydrophobic pocket predominantly formed by Met165, Leu167, and Gln192. This observation, along with the observed weaker electron density in this region (Fig. 3e), suggests that this subsite could be modified for an improved inhibitor.

In solving the complex of compound 4 with $3 \mathrm{CL}$ protease in both space groups $C 2$ and $P 1$, we observed that the $\mathrm{S1}^{\prime}$ site demonstrated conformational flexibility (Fig. 4c). In addition, the S4 site demonstrated weaker electron density (Fig. 3d), suggesting that modifying the interaction of compound 4 with these two subsites could improve the compound's inhibitory activity.

The finding that MAC-5576 was covalently linked to Cys145 in the crystal structure (Fig. 3c) but did not display time-dependent inhibition (Fig. 2c) suggests that it may be a reversible covalent inhibitor. The overall weaker electron density and optimal modeling with occupancy set to 0.5 for this structure supports the possibility of its reversible nature. However, it is possible that the lack of time-dependent inhibition, yet the observation of a clear covalent linkage in the crystal structure, is due to the differences in the conditions used for the two experiments. Further investigations into the mechanism of action of MAC-5576 may reveal a method for alleviating its lack of activity in inhibiting the virus (Fig. 2d).

The collective observations from the three inhibitors suggest that development of 3CL protease inhibitors may benefit from first establishing robust interactions within the S1, S2, and/or S1 ${ }^{\prime}$ sites, before extending into the S3 and S4 sites. For these, and other compounds targeting the 3CL protease, there are ample opportunities to improve the inhibitory potencies against the $3 \mathrm{CL}^{\text {pro }}$ by designing compounds that exploit the accessible contact points to strengthen the ligand-protein interactions (Fig. 4d).

In summation, we have identified compound 4, GC376, and MAC-5576 as inhibitors of the SARS-CoV-2 3CL protease. Crystal structures of the compounds complexed to the protease suggested their mechanisms of action, as well as portended guidelines for the development of SARS-CoV-2 3CL protease inhibitors, which may aid in the future development of novel inhibitors to combat this virus.

\section{Methods}

Compounds. Compound 4 was synthesized using the synthesis route previously described, with the exception of using a sodium borohydride-cobaltous chloride 
a

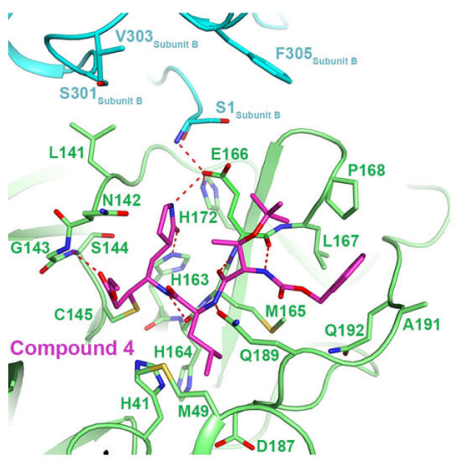

d

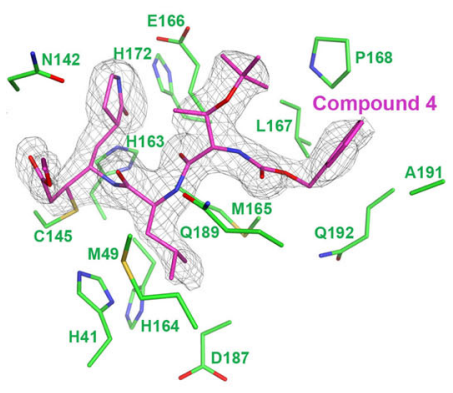

b

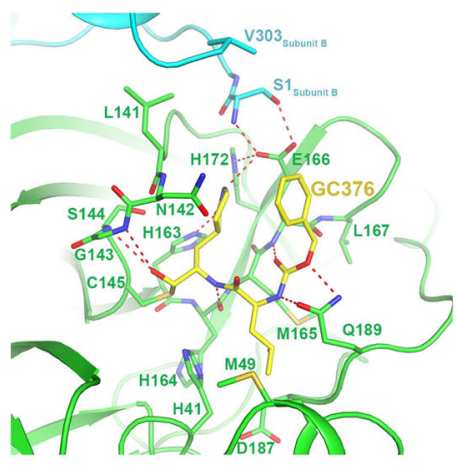

e

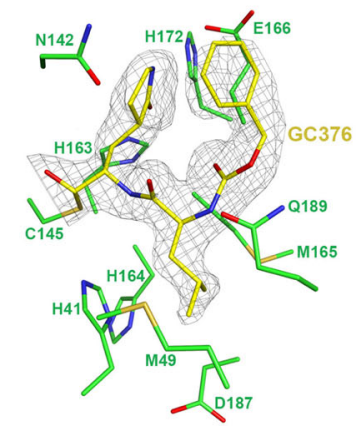

C

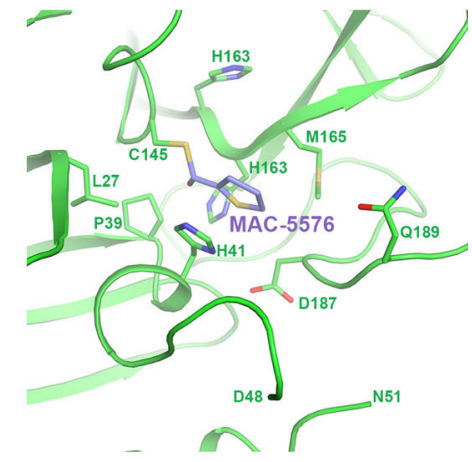

f

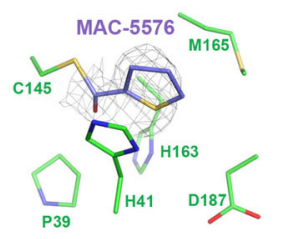

Fig. 3 Crystal structures of inhibitors in complex with the SARS-CoV-2 3CL protease. Structure of compound 4 (a), GC376 (b), or MAC-5576 (c) bound to SARS-CoV-2 3CLpro. Protomer A is denoted in green and protomer B is denoted in cyan. The Fo-Fc omit map, contoured at $3 \sigma$ (gray), for compound 4 (d), GC376 (e), and MAC-5576(f).

reduction of the nitrile in the construction of the lactam, thus avoiding the high pressure hydrogenation in the original route ${ }^{7,13}$. GC376 was purchased from Aobious (Gloucester, MA, USA) and MAC-5576 was purchased from Maybridge (Cheshire, United Kingdom).

Expression and purification of SARS-CoV-2 3CL protease. The SARS-CoV-2 $3 \mathrm{CL}$ protease gene was codon optimized for bacterial expression and synthesized (Supplementary Table 1) (Twist Bioscience, San Francisco, CA, USA), then cloned into a bacterial expression vector (pGEX-5X-3, GE, Boston, MA, USA, gift from Yosef Sabo, Columbia University Irving Medical Center) which expresses the protease as a fusion construct with a N-terminal GST tag, followed by a Factor Xa cleavage site (pGEX-5X-3-SARS-CoV-2-3CL, deposited to Addgene as plasmid \#168457). After confirmation by Sanger sequencing using the primers listed in Supplementary Table 2, the construct was transformed into BL21 (DE3) cells. These E. coli were inoculated and grown overnight as starter cultures, then used to inoculate larger cultures at a 1:100 dilution, which were then grown at $37^{\circ} \mathrm{C}, 220$ RPM until the OD reached 0.6-0.7. Expression of the protease was induced with the addition of $0.5 \mathrm{mM}$ IPTG, and then the cultures were incubated at $16^{\circ} \mathrm{C}, 180$ RPM for $10 \mathrm{~h}$. Cells were pelleted at $3580 \times g$ for $15 \mathrm{~min}$ at $4^{\circ} \mathrm{C}$, resuspended in lysis buffer $(20 \mathrm{mM}$ Tris- $\mathrm{HCl}, \mathrm{pH} 8.0,300 \mathrm{mM} \mathrm{NaCl})$, homogenized by sonication, then clarified by centrifuging at $25,000 \times g$ for $1 \mathrm{~h}$ at $4{ }^{\circ} \mathrm{C}$. The supernatant was mixed with Glutathione Sepharose resin (Sigma, St. Louis, MO, USA) and placed on a rotator for $2 \mathrm{~h}$ at $4{ }^{\circ} \mathrm{C}$. The resin was then repeatedly washed by centrifugation at $3210 \times g$ for $15 \mathrm{~min}$ at $4{ }^{\circ} \mathrm{C}$, discarding of the supernatant, and then resuspension of the resin in fresh lysis buffer. After ten washes, the resin was resuspended in lysis buffer, and Factor Xa was added and incubated for $36 \mathrm{~h}$ at $4^{\circ} \mathrm{C}$ on a rotator. The resin was centrifuged at $3210 \times g$ for $15 \mathrm{~min}$ at $4{ }^{\circ} \mathrm{C}$, and then the supernatant was collected and concentrated using a $10 \mathrm{kDa}$ concentrator before being loaded onto a Superdex 10/300 GL column in $50 \mathrm{mM}$ Tris- $\mathrm{HCl}, \mathrm{pH}$ 7.5, $1 \mathrm{mM}$ EDTA for further purification by size exclusion chromatography. The appropriate fractions were collected and pooled with a $10 \mathrm{kDa}$ concentrator, and then the final product was assessed for quality by SDS-PAGE and measurement of biochemical activity.

Measurement of SARS-CoV-2 3CL protease biochemical activity. The in vitro biochemical activity of the SARS-CoV-2 3CL protease was measured as previously described $^{5}$. The fluorogenic peptide MCA-AVLQSGFR-Lys(DNP)-Lys-NH2, corresponding to the nsp4/nsp5 cleavage site in the virus, was synthesized (GL Biochem, Shanghai, China), then resuspended in DMSO to use as the substrate. Different concentrations of this substrate, ranging from 5 to $100 \mu \mathrm{M}$, were prepared in the assay buffer ( $50 \mathrm{mM}$ Tris- $\mathrm{HCl}, \mathrm{pH} 7.5,1 \mathrm{mM}$ EDTA) in a 96 well-plate. The protease was then added to each well at a concentration of $0.2 \mu \mathrm{M}$, and then fluorescence was continuously measured on a plate reader for $3 \mathrm{~min}$. The catalytic efficiency of the protease was then calculated by nonlinear regression (GraphPad Prism, GraphPad Software, San Diego, CA, USA). For calculations, a 100\% active enzyme was assumed.

Measurement of SARS-CoV-2 3CL protease inhibition. Inhibition of the biochemical activity of the SARS-CoV-2 3CL protease was quantified as previously described with modifications ${ }^{5}$. Serial dilutions of the test compound were prepared in the assay buffer, and then incubated with $0.2 \mu \mathrm{M}$ of the protease for $10 \mathrm{~min}$ at $37^{\circ} \mathrm{C}$. The substrate was then added at $20 \mu \mathrm{M}$ per well, and then fluorescence was continuously measured on a plate reader for $3 \mathrm{~min}$. Inhibition was then calculated by comparison to control wells with no inhibitor added. $\mathrm{IC}_{50}$ values were determined by nonlinear regression (GraphPad Prism). For calculations, a $100 \%$ active enzyme was assumed.

Kinetic parameters were determined as previously described ${ }^{14}$. Compounds were pre-incubated with the protease at differing timepoints at various concentrations to derive $k_{\text {obs }}$, which were then used for the calculation of $k_{\text {inact }}$ and $K_{\mathrm{i}}$ by nonlinear regression (GraphPad Prism).

Measurement of SARS-CoV-2 viral inhibition. Stocks of SARS-CoV-2 strain 2019-nCoV/USA_WA1/2020 were propagated and titered in Vero-E6 cells. One day prior to the experiment, Vero-E6 cells were seeded at 30,000 cells/well in 96 well-plates. Serial dilutions of the test compound were prepared in cell media (EMEM $+10 \%$ FCS + penicillin/streptomycin), overlaid onto cells, and then virus was added to each well at an MOI of 0.2 . Cells were incubated at $37^{\circ} \mathrm{C}$ under $5 \%$ $\mathrm{CO}_{2}$ for $72 \mathrm{~h}$ and then viral cytopathic effect was scored in a blinded manner. Inhibition was calculated by comparison to control wells with no inhibitor added. $\mathrm{EC}_{50}$ values were determined by nonlinear regression (GraphPad Prism). Cells were confirmed as mycoplasma negative prior to use. All experiments were conducted in a biosafety level 3 (BSL-3) lab.

Measurement of cellular cytotoxicity. Vero-E6 cells were incubated with the compound of interest for $48 \mathrm{~h}$ at $37^{\circ} \mathrm{C}$ under $5 \% \mathrm{CO}_{2}$ and then cellular cytotoxicity was determined with the XTT Cell Proliferation Assay Kit (ATCC) according to the manufacturer's instructions. 
a

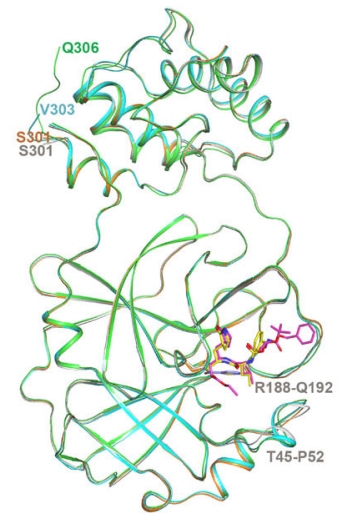

d b

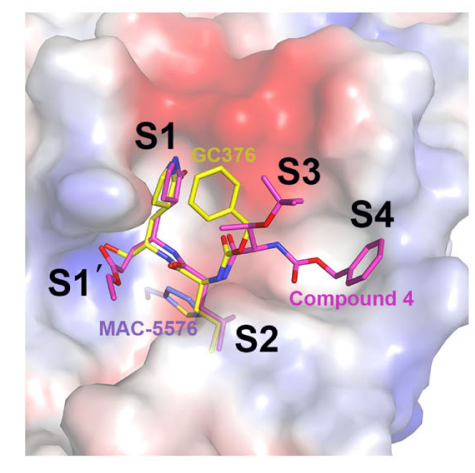

C

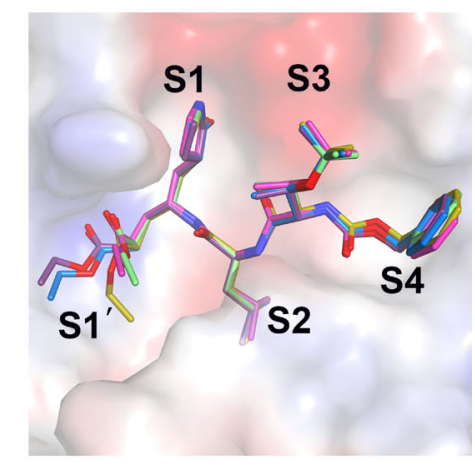

e
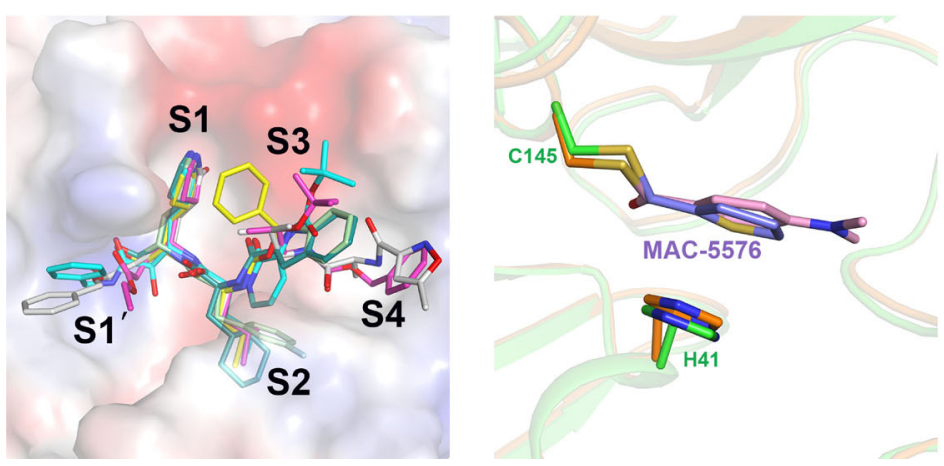

Fig. 4 Overlays of the crystal structures. a Crystal structure of the ligand-free $3 C_{L}$ pro (gray), in complex with, compound 4 (green for $3 C L^{p r o}$ and magenta for compound 4), GC376 (cyan for 3CL pro and yellow for GC376), and MAC-5576 (orange for 3CL pro and purple for MAC-5576). One protomer for each structure is shown, with the inhibitors shown with stick models. The terminal residue of each structure, as well as two stretches of residues near the binding site that exhibit local conformational change between the ligand-free and inhibitor-bound structures are labeled. $\mathbf{b}$ Overlay of all three compounds in the substrate-binding pocket of the $3 \mathrm{CL}$ protease. c Overlay of five molecules of compound 4, one from space group $C 2$ (magenta) and four from four promoters in space $P 1$ (light green, purple, marine, dark gold) for compound 4 bound to promoter A, B, C, and D of space group P1, respectively. d Comparison of the binding modes of compound 4 and GC376 with other peptide-like inhibitors. Compound 4 (magenta) and GC376 (yellow) were overlaid with previously identified compounds, compound 13b (cyan, PDB: 6Y2F), compound 11a (dark green, PDB: 6LZE), compound 11b (light green, PDB: $6 \mathrm{MOK}$ ), and N3 (white; PDB: 7BQY). e Comparison of the binding modes of MAC-5576 with XP-59. MAC-5576 (purple) bound to the SARS-CoV-2 $3 C L$ protease (orange) was overlaid with XP-59 (pink) bound to the SARS-CoV 3CL protease (green, PDB: 2V6N).

Crystallization, data collection, and structure determination. To generate the complex of SARS-CoV-2 $3 \mathrm{CL}$ protease bound to compound 4, $50 \mu \mathrm{M}$ of the $3 \mathrm{CL}$ protease was incubated with $500 \mu \mathrm{M}$ of compound 4 in a buffer comprised of $50 \mathrm{mM}$ Tris- $\mathrm{HCl}(\mathrm{pH} 7.5), 1 \mathrm{mM}$ EDTA, and $5 \%(\mathrm{v} / \mathrm{v})$ glycerol for $1 \mathrm{~h}$ at $4{ }^{\circ} \mathrm{C}$. This complex was then concentrated to $8.5 \mathrm{mg} / \mathrm{mL}$ using a $10 \mathrm{kDa}$ concentrator, and initially subjected to extensive robotic screening at the High-Throughput Crystallization Screening Center of the Hauptman-Woodward Medical Research Institute (HWI) (https://hwi.buffalo.edu/high-throughput-crystallization-center/) ${ }^{15}$. The most promising crystal hits were then reproduced using the microbatch-under-oil method at $4{ }^{\circ} \mathrm{C}$. Block-like crystals of $3 \mathrm{CL}^{\text {pro }}$ in complex with compound 4 appeared after a few days in the crystallization condition comprised of $0.1 \mathrm{M}$ potassium nitrate, $0.1 \mathrm{M}$ sodium acetate ( $\mathrm{pH}$ 5), and $20 \%(\mathrm{w} / \mathrm{v})$ PEG 1000 with protein to crystallization reagent at a 2:1 ratio. The crystals were subsequently transferred into the same crystallization reagent supplemented with $15 \%(\mathrm{v} / \mathrm{v})$ glycerol and flashfrozen in liquid nitrogen. Plate-like crystals of $3 \mathrm{CL}^{\text {pro }}$ in complex with compound 4 were also produced using crystallization reagent comprising $0.1 \mathrm{M}$ Bis-Tris ( $\mathrm{pH} 6.5$ ) and 20\% (w/v) PEG MME 5000.

To obtain crystals of $3 \mathrm{CL}^{\text {pro }}$ in complex with GC376, crystals of ligand-free $3 \mathrm{CL}^{\text {pro }}$ were initially grown by using seeding method in a crystallization reagent comprised of $0.1 \mathrm{M}$ sodium phosphate-monobasic, $0.1 \mathrm{M} \mathrm{MES} \mathrm{(pH} \mathrm{6),} \mathrm{and} \mathrm{20 \%}$ (w/v) PEG 4000. These crystals were subsequently soaked with $15 \mathrm{mM} \mathrm{GC376,}$ followed by flash-freezing of the crystals in the same reagent supplemented with $15 \%$ ethylene glycol.

To generate the complex of SARS-CoV-2 3CL protease bound to MAC-5576, $50 \mu \mathrm{M}$ of the $3 \mathrm{CL}$ protease was incubated with $500 \mu \mathrm{M}$ of MAC-5576 in a buffer comprised of $50 \mathrm{mM}$ Tris- $\mathrm{HCl}(\mathrm{pH} 7.5), 1 \mathrm{mM}$ EDTA, and $5 \%(\mathrm{v} / \mathrm{v})$ glycerol for $1 \mathrm{~h}$ at $4{ }^{\circ} \mathrm{C}$. The complex was concentrated to $10 \mathrm{mg} / \mathrm{mL}$ using a $10 \mathrm{kDa}$ concentrator, and then crystallized in the same conditions as those used for crystallization of ligand-free $3 \mathrm{CL}^{\text {pro }}$.
A native dataset was collected on each crystal of $3 \mathrm{CL}^{\text {pro }}$, alone (ligand-free), and in complex with compound 4 and GC376 at the NE-CAT24-ID-C beam line of Advanced Photon Source (APS) at Argonne National Laboratory, and the NE-CAT 24-ID-E beam line of APS was used for data collection on crystals of 3CL-MAC5576. Crystals of ligand-free $3 \mathrm{CL}^{\text {pro }}$ and in complex with compound 4 in space group $C 2$ and $P 1$, GC376, and MAC-5576 diffracted the X-ray beam to resolution $1.85,1.94,1.84,1.83,1.73 \AA$, respectively. The images were processed and scaled in space group $C 2$ using $\mathrm{XDS}^{16}$. The structure of $3 \mathrm{CL}^{\text {pro }}$ with compound 4 in space group $C 2$ was determined by molecular replacement (MR) method using program MOLREP ${ }^{17}$ and the crystal structure of $3 \mathrm{CL}^{\text {pro }}$ in complex with inhibitor N3 (PDB id: $6 \mathrm{LU} 7)^{5}$ was used as a search model. The structure of $3 \mathrm{CL}^{\text {pro }}$ with compound 4 in space group $P 1$ was also determined by MR method and the refined model of $3 \mathrm{CL}^{\text {pro }}$ with compound 4 in space group $C 2$ was used as the search model. The geometry of each crystal structure was subsequently fixed and the corresponding inhibitor was modeled in by XtalView ${ }^{18}$ and $\operatorname{Coot}^{19}$, and refined using PHENIX ${ }^{20}$. The mapping of electrostatic potential surfaces was generated in PyMOL with the APBS plug-in ${ }^{21}$. There is one protomer of $3 \mathrm{CL}^{\text {pro }}$ complex in the asymmetric unit of each crystal of space group $C 2$, and there are four protomers of $3 \mathrm{CL}^{\text {pro }}$ bound to compound 4 in each unit cell of space group $P 1$. The crystallographic statistics are shown in Table 1.

Reporting summary. Further information on experimental design is available in the Nature Research Reporting Summary linked to this paper.

\section{Data availability}

The SARS-CoV-2 $3 \mathrm{CL}^{\text {pro }}$ bacterial expression vector utilized in this study has been deposited to Addgene as plasmid \#168457. Structural data for the ligand-free SARS-CoV-2 $3 \mathrm{CL}$ protease and $3 \mathrm{CL}^{\mathrm{pro}}$ in complex with compound 4 in space group $C 2$ and P1, GC376, 
and MAC-5576 have been deposited in the Protein Data Bank (PDB) under accession codes 7JST, 7JT7, 7JW8, 7JSU, and 7JT0, respectively. Overlays in Fig. 4d, e were made using previously deposited structures in PDB, available under accession codes 6Y2F (SARS-CoV-2 $3 \mathrm{CL}^{\text {pro }}$ with compound 13b), 6LZE (SARS-CoV-2 3CL pro with compound 11a), 6M0K (SARS-CoV-2 3CLpro with compound 11b), 7BQY (SARS-CoV-2 3CLpro with N3), and $2 \mathrm{~V} 6 \mathrm{~N}$ (SARS-CoV $3 \mathrm{CL}^{\text {pro }}$ with XP-59). Source data are provided with this paper.

Received: 7 July 2020; Accepted: 10 March 2021;

Published online: 01 April 2021

\section{References}

1. $\mathrm{Wu}, \mathrm{F}$. et al. A new coronavirus associated with human respiratory disease in China. Nature 579, 265-269 (2020).

2. Zhou, P. et al. A pneumonia outbreak associated with a new coronavirus of probable bat origin. Nature 579, 270-273 (2020).

3. Beigel, J. H. et al. Remdesivir for the Treatment of Covid-19-Preliminary Report. N. Engl. J. Med. https://doi.org/10.1056/NEJMoa2007764 (2020).

4. Wang, Y. et al. Remdesivir in adults with severe COVID-19: a randomised, double-blind, placebo-controlled, multicentre trial. Lancet 395, 1569-1578 (2020).

5. Jin, Z. et al. Structure of M(pro) from SARS-CoV-2 and discovery of its inhibitors. Nature, https://doi.org/10.1038/s41586-020-2223-y (2020).

6. Zhang, L. et al. Crystal structure of SARS-CoV-2 main protease provides a basis for design of improved alpha-ketoamide inhibitors. Science 368, 409-412 (2020).

7. Yang, S. et al. Synthesis, crystal structure, structure-activity relationships, and antiviral activity of a potent SARS coronavirus 3CL protease inhibitor. J. Med. Chem. 49, 4971-4980 (2006).

8. Kim, Y. et al. Broad-spectrum antivirals against 3C or 3C-like proteases of picornaviruses, noroviruses, and coronaviruses. J. Virol. 86, 11754-11762 (2012).

9. Blanchard, J. E. et al. High-throughput screening identifies inhibitors of the SARS coronavirus main proteinase. Chem. Biol. 11, 1445-1453 (2004).

10. Dai, W. et al. Structure-based design of antiviral drug candidates targeting the SARS-CoV-2 main protease. Science https://doi.org/10.1126/science.abb4489 (2020).

11. Verschueren, K. H. et al. A structural view of the inactivation of the SARS coronavirus main proteinase by benzotriazole esters. Chem. Biol. 15, 597-606 (2008).

12. Ma, C. et al. Boceprevir, GC-376, and calpain inhibitors II, XII inhibit SARSCoV-2 viral replication by targeting the viral main protease. Cell Res. 30, 678-692 (2020).

13. Zhai, Y. et al. Cyanohydrin as an anchoring group for potent and selective inhibitors of enterovirus 71 3C protease. J. Med. Chem. 58, 9414-9420 (2015).

14. Obach, R. S., Walsky, R. L. \& Venkatakrishnan, K. Mechanism-based inactivation of human cytochrome p450 enzymes and the prediction of drugdrug interactions. Drug Metab. Dispos. 35, 246-255 (2007).

15. Luft, J. R. et al. A deliberate approach to screening for initial crystallization conditions of biological macromolecules. J. Struct. Biol. 142, 170-179 (2003).

16. Kabsch, W. Integration, scaling, space-group assignment and post-refinement. Acta. Crystallogr. D. Biol. Crystallogr. 66, 133-144 (2010).

17. Vagin, A. \& Teplyakov, A. Molecular replacement with MOLREP. Acta. Crystallogr. D. Biol. Crystallogr. 66, 22-25 (2010).

18. McRee, D. E. XtalView/Xfit-A versatile program for manipulating atomic coordinates and electron density. J. Struct. Biol. 125, 156-165 (1999).

19. Emsley, P., Lohkamp, B., Scott, W. G. \& Cowtan, K. Features and development of Coot. Acta. Crystallogr. D. Biol. Crystallogr. 66, 486-501 (2010).

20. Adams, P. D. et al. PHENIX: a comprehensive Python-based system for macromolecular structure solution. Acta. Crystallogr. D. Biol. Crystallogr. 66, 213-221 (2010).

21. Baker, N. A., Sept, D., Joseph, S., Holst, M. J. \& McCammon, J. A. Electrostatics of nanosystems: application to microtubules and the ribosome. Proc. Natl Acad. Sci. USA 98, 10037-10041 (2001).

\section{Acknowledgements}

This work was supported by a grant from the Jack Ma Foundation to D.D.H. and A.C and by grants from Columbia Technology Ventures and the Columbia Translational Therapeutics (TRx) program to B.R.S. A.C. is also supported by a Career Awards for Medical Scientists from the Burroughs Wellcome Fund. S.I. was supported by NIH grant T32AI106711. We are thankful to Yosef Sabo for the pGEX-5X-3 plasmid, to WuXi AppTec for assistance with the enzyme kinetics assay, to the staff of the HighThroughput Crystallization Screening Center of the Hauptman-Woodward Medical Research Institute for screening of crystallization conditions, and to the staff of the Advanced Photon Source at Argonne National Laboratory for assistance with data collection. Crystallization screening was supported through NSF grant 2029943. This research used resources of the Advanced Photon Source, a U.S. Department of Energy (DOE) Office of Science User Facility, operated for the DOE Office of Science by Argonne National Laboratory under Contract No. DE-AC02-06CH11357. Extraordinary facility operations were supported in part by the DOE Office of Science through the National Virtual Biotechnology Laboratory, a consortium of DOE national laboratories focused on the response to COVID-19, with funding provided by the Coronavirus CARES Act. Part of the research was conducted at the Northeastern Collaborative Access Team beamlines, which are funded by the National Institute of General Medical Sciences from the National Institutes of Health (P30 GM124165).

\section{Author contributions}

D.D.H. conceived the project. S.I., B.R.S., A.C., and D.D.H. planned and designed the experiments. S.I. and S.J.H. cloned, expressed, and purified proteins. A.Z. synthesized compound 4. S.I. conducted the protease inhibition assay. M.N. and Y.H. conducted the antiviral assay and cytotoxicity measurements. F.F. and H.L. crystallized the proteins. F.F. collected diffraction data and solved the crystal structures. F.F., F.-Y.L., and L.X. analyzed the structural data. S.I., F.F., F.-Y.L., L.X., A.C., and D.D.H. wrote the manuscript with input from all authors.

\section{Competing interests}

S.I., F.F., H.L., A.Z., B.R.S., A.C., and D.D.H. are inventors on a patent application submitted based on this work. B.R.S. is an inventor on additional patents and patent applications related to small molecule therapeutics, and co-founded and serves as a consultant to Inzen Therapeutics and Nevrox Limited. The other authors have no competing interests.

\section{Additional information}

Supplementary information The online version contains supplementary material available at https://doi.org/10.1038/s41467-021-22362-2.

Correspondence and requests for materials should be addressed to B.R.S., A.C. or D.D.H

Peer review information Nature Communications thanks the anonymous reviewer(s) for their contribution to the peer review of this work.

Reprints and permission information is available at http://www.nature.com/reprints

Publisher's note Springer Nature remains neutral with regard to jurisdictional claims in published maps and institutional affiliations.

\footnotetext{
Open Access This article is licensed under a Creative Commons Attribution 4.0 International License, which permits use, sharing, adaptation, distribution and reproduction in any medium or format, as long as you give appropriate credit to the original author(s) and the source, provide a link to the Creative Commons license, and indicate if changes were made. The images or other third party material in this article are included in the article's Creative Commons license, unless indicated otherwise in a credit line to the material. If material is not included in the article's Creative Commons license and your intended use is not permitted by statutory regulation or exceeds the permitted use, you will need to obtain permission directly from the copyright holder. To view a copy of this license, visit http://creativecommons.org/ licenses/by/4.0/
}

(C) The Author(s) 2021, corrected publication 2021 